\title{
Tophaceous Gout of Hip Joint Mimicking Bone Tumor
}

\author{
I. Mujaddid*, E. Rosihan, T. H. Handry, W. Brian
}

Department of Orthopedic and Traumatology, Faculty of Medicine, Universitas Sebelas Maret, Prof. Dr. R. Soeharso Orthopedic Hospital, Solo, Indonesia

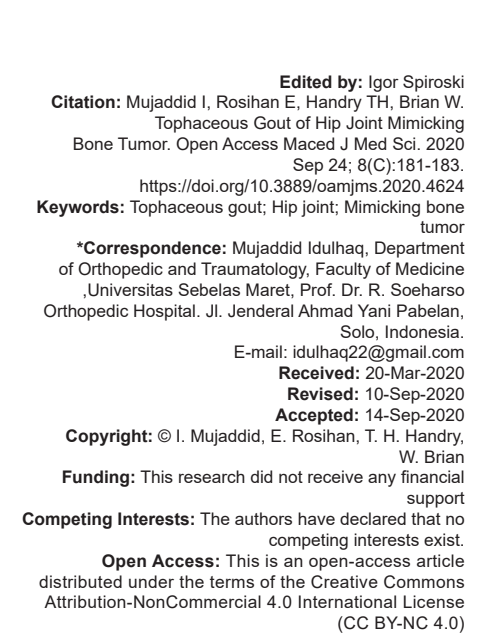

Introduction

Gout is a common metabolic disease characterized by elevated serum urate concentration, acute arthritis, deposition of monosodium urate monohydrate crystal (tophus), mainly around joints, and renal disease. It is typically a disease of adult males, with a peak age at onset in the fifth decade of life [1]. Four stages of gout have recognized, among others are asymptomatic hyperuricemia, acute gouty arthritis, polyarticular gouty arthritis, and chronic tophaceous gout. Atypical presentations of gout occurring in young male or female patients have attributed to alcoholism, accelerated purine nucleotide degradation, genetic predisposition, and congenital diseases such as Lesch Nyhan syndrome [2]. Gout is known as a "great mimicker" since ancient Greek times when Hippocrates attempted to differentiate it from rheumatoid arthritis. It reported mimicking joint and soft-tissue infections, skin malignancies, nerve compression syndromes, and soft-tissue tumors. Gouty tophi often found in the foot around the big toe as well as the ankle region [3].

\section{Case Presentation}

A 49-year-old woman was referred to our orthopedic oncology department with pain and swelling over her right hip for 3 years. There was no history of preceding trauma, pain at the other joint, and gout neither in the patient nor her family. The pain progressively worsened over the past 4 months. It exacerbated when she tried to flex her hip and when she walked. Associated with the pain, she noticed a lump over the right hip, which was visible while she flexed and internally rotated her right hip. There was no history of fever, loss of appetite, or loss of weight.

Physical examination revealed a lump at the posterolateral aspect of the right hip, above the greater trochanter, sized $3 \mathrm{~cm} \times 4 \mathrm{~cm}$. It was dense and immobile. There were no skin changes or inflammation signs. The patients experienced a limited range of movement due to the pain in her right hip.

Laboratory findings showed a typical full blood picture. The patient's erythrocyte sedimentation rate was $11 \mathrm{~mm} / \mathrm{h}$. The patient total white count was 
$7300 / \mathrm{mm}^{3}$. A plain radiograph of the pelvic showed the opacity on the posterior aspect femoral neck. It is suggesting a benign bone tumor suspicious of osteochondroma [Figure 1]. CT Scan of pelvic showed

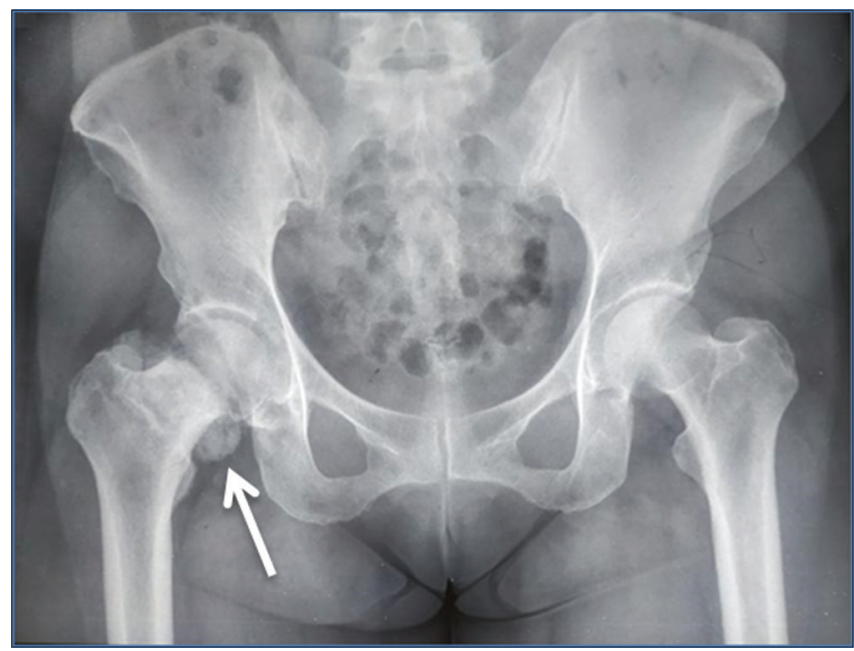

Figure 1: Opacity around femoral neck suggesting benign bone tumor

a hyperdense lesion at posterior aspect femoral neck sized $3.85 \mathrm{~cm} \times 5.04 \mathrm{~cm} \times 3.23 \mathrm{~cm}$, lobulated shaped with well-defined border and irregular shaped. There was no extent to surrounding soft-tissue, droved suggestion for benign tumors (Figure 2). MRI unperformed due to CT results already directing to the benign lesion (osteochondroma).
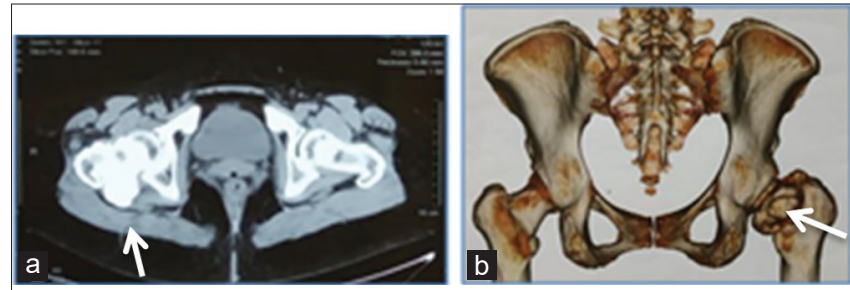

Figure 2: ( $a$ and b) Computed tomography scan revealing a benign bone tumor of the right hip

On the assumption of a benign bone tumor such as osteochondroma, the patient underwent surgery. At operation, the expanded mass of the posterior neck femur $3 \times 3 \times 2 \mathrm{~cm}$ in size. It was found enveloped with a capsule and contained chalky white content [Figure 3]. The pathological report was that of a gouty tophus [Figure 4] and radiological result post operation in Figure 5.

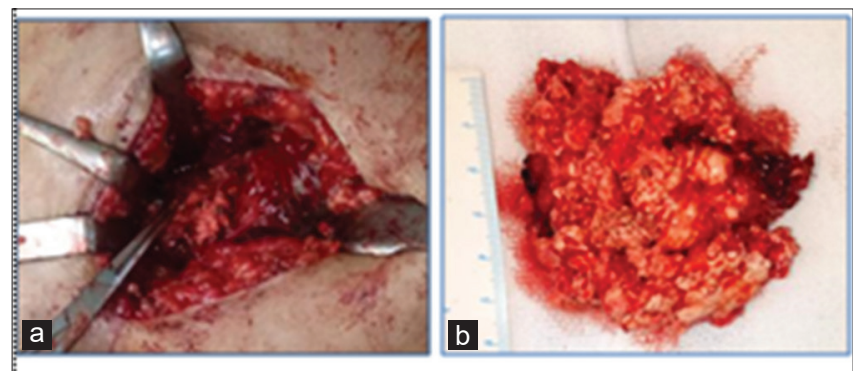

Figure 3: Intraoperative view showing the tumor mass situated within the capsule hip joint which appears to be well encapsulated (left); after resection, the tumor was chalky white (right)

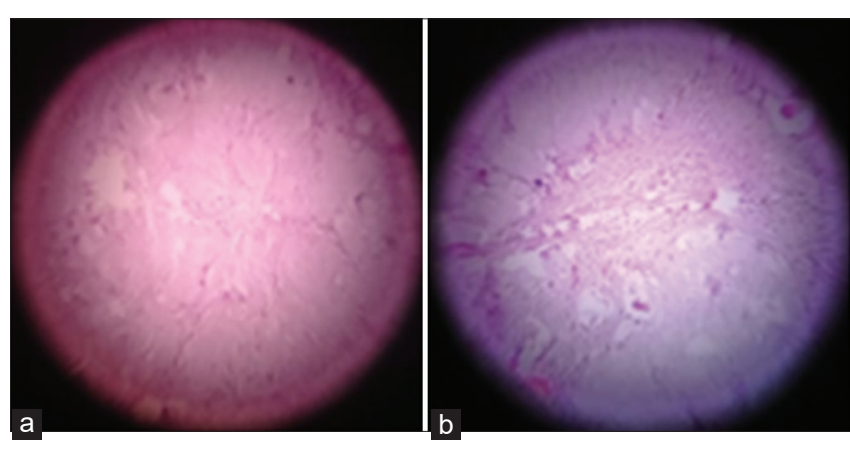

Figure 4: Pathology of tumor shows focus calcification surrounded by granulated.tissue, macrophage, and mononuclear cells

During hospitalization, there was complex assessment among others are clinical and laboratory evaluations, the previous disease and family history, blood pressure, complete blood count, platelet, serum uric acid, cholesterol, triglyceride levels, blood urea nitrogen, and creatinine levels. There is no evidence of hypertension, renal disease, or diabetes mellitus. No predisposing factors such as alcoholism traced. After discharge, the patient has had follow-up at the outpatient department. There was no underlying systemic disorder found yet. It is considered the diagnosis of primary idiopathic tophaceous gout. The patient no longer felt pain at 1 year of follow-up.

\section{Discussion}

Gout is a condition resulting from an overload of uric acid crystals that deposit in tissues of the body and features recurring attacks of acute joint inflammation. Chronic gout can lead to deposits of hard lumps of uric acid in and around the joints and tendons. Gout has known to mimic such diverse conditions as joint and soft-tissue infections, nerve compression, and even soft-tissue tumors [2]. Gout usually involves inferior limbs and particularly the first metatarsophalangeal joint, ankles, and knees. The diagnosis may be confirmed

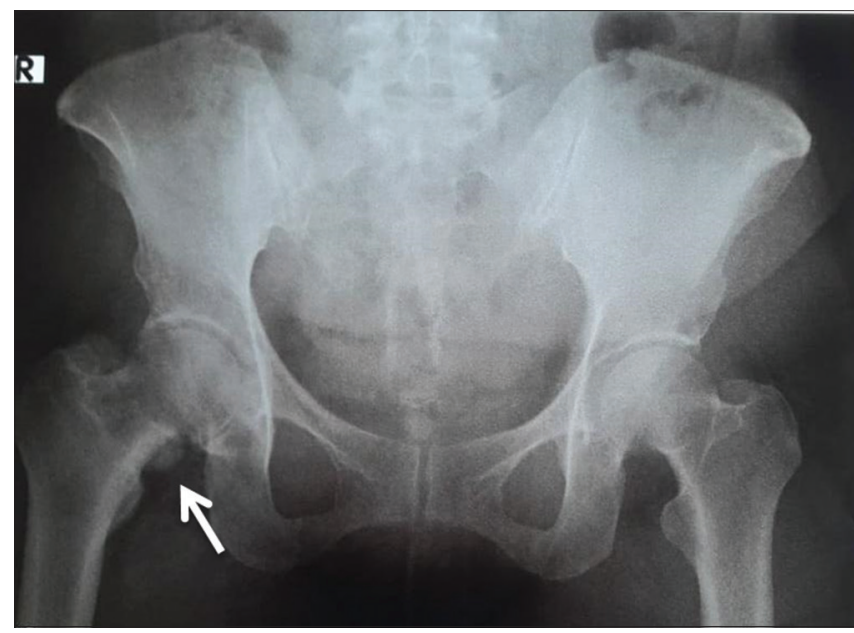

Figure 5: Post-operative radiograph 
by identification of monosodium urate crystals in the synovial fluid of the affected joint. Acute gout may be treated with nonsteroidal anti-inflammatory drugs, corticosteroids, or colchicine diagnosis, treatment, and prevention of gout [4].

Hip localization of gout is uncommon. To the best of our knowledge, only few reports of gouty arthritis affecting the hip, with a documented presence of urate crystal, have been described [5]. Fua and Lee reported the prevalence of patients with acute gout with normal uric acid levels at diagnosis to be $12 \%$. In a review of diagnostic criteria for gout, crystal identification in the synovial fluid remains the gold standard for the identification of gout [6].

This case illustrates the variable presentation of gout. The diagnosis of any soft-tissue swelling such as gout is not always easy, specifically if it occurs in an unusual place [7]. The post-operative radiograph showed opacity existing picture, which probably obtained when tumor wall mass not taken by surgery [6]. Clinically, there is no relapse of pain after the operation.

The hip is a deep and less accessible joint. A hip joint aspiration requires considerable expertise and resources such as an ultrasound or CT for image guidance. The use of ultrasound-guided joint aspiration has reported in West Africa for a case of acute gout of the hip joint. Ultrasound visualization for joint aspiration poses less risk to patients with pre-existing renal impairment compared to the use of CT [8].

\section{Conclusions}

Tophaceous gout in the specific location of the hip joint is a rare case. Because of its mimicking, clinical and radiology assessments can mislead the interpretation. Tophaceous gout in the hip joint detected as bone tumors with its characteristics. Furthermore, it requires a better application and diagnosed for the mimicked tophaceous. The use of ultrasound-guided joint aspiration reported as a better diagnosis for gout in rare areas.

\section{References}

1. Zhang B, Fang W, Zeng X, Zhang Y, Ma Y, Sheng F, et al. Clinica characteristics of early-and late-onset gout a cross-sectional observational study from a Chinese gout clinic. Medicine (Baltimore). 2016;95(47):e5425. https://doi.org/10.1097/ md.0000000000005425

PMid:27893683

2. Sakti M, Usman MA, Lee J, Benjamin M, Maulidiah Q. Atypical musculoskeletal manifestations of gout in hyperuricemia patients. Open Access Rheumatol Res Rev 2019;11:47-52. https://doi.org/10.2147/oarrr.s192369 PMid:30799962

3. Zelen CM, Poka A, Andrews J. Foot and Ankle International. United States: SAGE Publications Inc.; 2013.

4. Hainer BL, Matheson E, Wilkes RT. Diagnosis, treatment, and prevention of gout. Am Fam Physician. 2014;90(12):831-6.

5. Tekaya R, Tekay AB, Saidane O, Said HB, Gaja A, Sahli H, et al. Tophaceous hip gouty arthritis revealing asymptomatic axial gout. Egypt Rheumatol. 2018;40(3):209-12. https://doi. org/10.1016/j.ejr.2017.10.001

6. Tobimatsu H, Nakayama M, Sakuma Y, Imamura H. Multiple tophaceous gout of hand with extensor tendon rupture. Case Rep Orthop. 2017;2017:1-4. https://doi.org/10.1155/2017/7201312

7. Fields TR. The challenges of approaching and managing gout. Rheum Dis Clin North Am. 2019;45(1):145-57. PMid:30447743

8. Fua TP, Dave Lee YH. Acute hip pain-a forgotten cause. Int J Clin Med 2016;7:177-81. https://doi.org/10.4236/ijcm.2016.73018 